\title{
On linear estimates with nearly minimum variance
}

\author{
By Gunnar Blom
}

\section{Introduction}

Let $z$ be a random variable with a continuous cumulative distribution function $F[(z-\mu) / \sigma]$ which depends upon two unknown parameters $\mu$ and $\sigma$. Consider an ordered random sample

$$
z_{(1)} \leqq z_{(2)} \leqq \cdots \leqq z_{(n)}
$$

of $z$-values. If the means and covariances of the reduced order statistics

$$
x_{i}=\frac{z_{(i)}-\mu}{\sigma} \quad(i=1,2, \ldots, n)
$$

are known, it is possible to find linear unbiased minimum variance estimates

$$
\sum_{i=1}^{n} g_{1 i} z_{(i)} \text { and } \sum_{i=1}^{n} g_{2 i} z_{(i)}
$$

of $\mu$ and $\sigma$ respectively (Lloyd, 1952). These estimates may be called best unbiased estimates. A serious drawback of the solution is that in most cases it involves very time-consuming numerical calculations.

The object of this paper is to show that, under general conditions, it is possible to find a convenient approximation to the best solution which may be termed a nearly best unbiased estimate. The variance of this estimate is, as some examples will show, often very little in excess of the minimum variance. The method presupposes that the means (but not the covariances) of the variables $x_{i}$ are known.

By a slight modification of the method it may be used also when neither the means nor the covariances are known. The resulting estimates will be called nearly best, nearly unbiased estimates.

Both types of estimates mentioned above may be derived from a theorem given in the next section.

\section{A theorem on linear estimates}

Denote by $E x_{i}$ and $\operatorname{cov}\left(x_{i}, x_{j}\right)$ the means and covariances of the variables $x_{i}(i=1,2, \ldots, n)$. Put $p_{i}=i /(n+1)$ and $q_{i}=1-p_{i}$. Further, define $\lambda_{i}=G\left(p_{i}\right)$, where $G(u)$ is the inverse function of $F(x)$. 
G. BLOM, Linear estimates with nearly minimum variance

Consider a linear estimate

$$
U=\sum_{i=1}^{n} g_{i} z_{(i)}
$$

of the parameter $k_{1} \mu+k_{2} \sigma$, where $k_{1}$ and $k_{2}$ are given constants.

Theorem. Let

$$
\theta_{0}, \theta_{1}, \ldots, \theta_{n}, \theta_{n+1}
$$

be any set of quantities subject to the conditions

$$
\theta_{0}=\theta_{n+1}=0 \quad \text { and } \quad \theta_{i} \neq 0 \quad(i=1,2, \ldots, n) .
$$

Replace the coefficients $g_{i}$ in (2.1) by new quantities

$$
h_{0}, h_{1}, \ldots, h_{n}
$$

defined, apart from an additive constant, by the relations

If the conditions

$$
g_{i}=\theta_{i}\left(h_{i}-h_{i-1}\right) \quad(i=1, \ldots, n) .
$$

$$
\sum_{i=0}^{n} C_{1 i} h_{i}=k_{1} ; \quad \sum_{i=0}^{n} C_{2 i} h_{i}=k_{2}
$$

are fultilled, where

$$
\dot{C}_{1 i}=\theta_{i}-\theta_{i+1} ; \quad C_{2 i}=\theta_{i} E x_{i}-\theta_{i+1} E x_{i+1} \quad(i=0,1, \ldots, n)
$$

then $U$ in (2.1) is an unbiased estimate of $k_{1} \mu+k_{2} \sigma$.

Further, the variance of $U$ can be written

where

$$
\operatorname{var} U=\frac{\sigma^{2}}{n+2}\left[\frac{1}{n+1} \sum_{i=0}^{n} h_{i}^{2}-\left(\frac{1}{n+1} \sum_{i=0}^{n} h_{i}\right)^{2}\right]+\sigma^{2} R
$$

$R_{i j}$ being defined by

$$
R=\sum_{i, j=1}^{n} g_{i} g_{j} R_{i}
$$

$$
\operatorname{cov}\left(x_{i}, x_{j}\right)=\frac{p_{i} q_{j}}{n+2}\left(\theta_{i} \theta_{j}\right)^{-1}+R_{i j} \quad(i \leqq j)
$$

The theorem holds for any choice of quantities $\theta_{i}$. It is, however, useful only when they are chosen so that $R$ in (2.5) behaves in a suitable way. In particular, it is desirable to choose $\theta_{i}$. so that $R$ tends rapidly to zero when $n$ increases. When $F(x)$ has a derivative $f(x)$ which is continuous (except, possibly, in the end-points of the range of variation), we take $\theta_{i}=f\left(\lambda_{i}\right)$. 
ARKIV För MATEMATIK. Bd $3 \mathrm{nr} 31$

\section{Derivation of the nearly best unbiased estimate}

The variance of $U$ given in (2.5) is, apart from the last term, proportional to

$$
Z=\sum_{i=0}^{n} h_{i}^{2}-\frac{1}{n+1}\left(\sum_{i=0}^{n} h_{i}\right)^{2} .
$$

Let now $Z$ be minimized with respect to the $h_{i}$ subject to the side conditions (2.3). The resulting quantities $h_{f}$ obviously provide an unbiased estimate of $k_{1} \mu+k_{2} \sigma$, but there is no guarantee that the true minimum of var $U$ is obtained, since $R$ is neglected. Determining $h_{i}$ in this way and using (2.2) we find as coefficients of the nearly best estimate

$$
g_{i}=\theta_{i}\left[a_{1}\left(C_{1 i}-C_{1 i-1}\right)+a_{2}\left(C_{2 i}-C_{2 i-1}\right)\right]
$$

where $a_{1}$ and $a_{2}$ are two multipliers. Introducing the notation

we have

$$
\begin{aligned}
d_{\mu \nu} & =\sum_{i=0}^{n} C_{\mu i} C_{\nu i} \quad(\mu, v=1,2), \\
D & =d_{11} d_{22}-d_{12}^{2}
\end{aligned}
$$

$$
\begin{aligned}
& a_{1}=\frac{1}{D}\left(k_{1} d_{22}-k_{2} d_{12}\right), \\
& a_{2}=\frac{1}{D}\left(-k_{1} d_{21}+k_{2} d_{11}\right) .
\end{aligned}
$$

When the distribution is symmetrical, $d_{12}=d_{21}=0$.

The coefficients of the nearly best estimates of $\mu$ and $\sigma$ are obtained by taking $k_{1}=1, k_{2}=0$ and $k_{1}=0, k_{2}=1$ respectively in these relations. It should be noticed that the solution depends upon the first and second order differences of the sequences $\left\{\theta_{i}\right\}$ and $\left\{\theta_{i} E x_{i}\right\}(i=0,1, \ldots, n+1)$.

The nearly best estimates of $\mu$ and $\sigma$ have been determined in the case $n=5$ for some special functions $F(x)$ for which best estimates are known. In these examples the quantities $\theta_{i}$ have been put equal to $f\left(\lambda_{i}\right)$.

As a measure of the efficiency of the estimates the quotient of the variance of the best estimate and the variance of the nearly best estimate has been used. (The last-mentioned quantity has been determined accurately by aid of existing tables, not from the approximation formula obtained by neglecting $R$ in (2.5).) The result of the calculations is given in Table 1. As seen from the table the loss of efficiency by using nearly best instead of best estimates is very small in these examples. (The estimate of $\mu$ in the normal case is of little interest but has been included for completeness.)

It should be mentioned that, in the rectangular case, the nearly best estimate is identical with the well-known best estimate based upon the extreme values of the sample.

When $F(x)$ has a continuous derivative which vanishes in the end-points of the range of variation, it may sometimes be convenient to replace the second 
G. BLOM, Linear estimates with nearly minimum variance

Table 1. Efficiency of nearly best linear estimates.

\begin{tabular}{|c|c|c|c|}
\hline \multirow{2}{*}{ Distribution } & \multicolumn{2}{|c|}{ Parameter } & \multirow{2}{*}{$\begin{array}{l}\text { Best estimate } \\
\text { given by }\end{array}$} \\
\hline & $\mu$ & $\sigma$ & \\
\hline 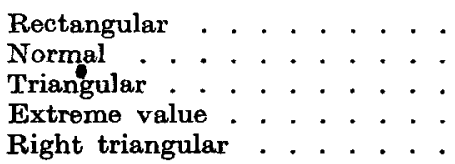 & $\begin{array}{c}100 \% \\
99.8 \\
94.4 \\
98.8 \\
99.9\end{array}$ & $\begin{array}{c}100 \% \\
99.7 \\
99.6 \\
96.6 \\
99.8\end{array}$ & $\begin{array}{l}\text { Lloyd } \\
\text { Godwin } \\
\text { Sarhan } \\
\text { Lieblein } \\
\text { Downton }\end{array}$ \\
\hline
\end{tabular}

Efficiency = quotient of variance of best linear umbiased estimate and variance of nearly best linear unbiased estimate.

order differences in (3.1) by the derivatives of $f(x)$. If this modification is used in the case of the normal distribution, the nearly best estimate of $\mu$ is replaced by the sample mean and the nearly best estimate of $\sigma$ by the estimate

$$
\frac{\sum_{i=1}^{n} \lambda_{i} z_{(i)}}{\sum_{i=1}^{n} \lambda_{i} E x_{i}} .
$$

The variance of this estimate is practically the same as that of the best estimate, the loss of efficiency being about $0.1 \%$ when $n=5$.

It deserves to be mentioned that the estimates obtained by the modified method are, apart from the values of the multipliers, identical with the estimates studied by Jung (1955) in the case of a class of distributions which, however, does not include the normal distribution.

\section{Nearly best, nearly unbiased estimates}

When the means of the variables $x_{i}$ are unknown, the approximation

$$
E x_{i} \sim G\left(\frac{i-\alpha}{n-\alpha-\beta+1}\right)
$$

is used in the definition of $C_{2 i}$ in (2.4). In other respects the procedure is the same as in the preceding section. The resulting estimates will in general be biased. By a suitable choice of constants $\alpha$ and $\beta$ the bias may, however, often be very small. General rules for the selection of the constants may be formulated. The variance of the estimates obtained in this way is often practically the same as that of the nearly best unbiased estimate.

Finally it deserves to be mentioned that by a study of the asymptotic variance of nearly best estimates, it is possible to obtain information about the nature of estimates which are non-regular in the sense used by Cramér (1946, Ch. 32).

Details concerning the method described in this paper will be given in a fortheoming publication. 


\section{ARKIV FÖR MATEMATIK. Bd 3 nr 31}

\section{REFER E N C ES}

CramÉr, H. (1946), Mathematical Methods of Statistics. Princeton.

Downton, F. (1954), Least-squares estimates using ordered observations. Ann. Math. Stat. $25,303$.

GoDwIs, H. J. (1949), On the estimation of dispersion by linear systematie statisties. Biometrika 36,92 .

JUNG, J. (1955), On linear estimates defined by a continuous weight function. Arkiv för Matematik Bd 3 nr $15,199$.

Liebleiv, J. (1954), A new method of analyzing extreme-value data. Nat. Adv. Comm. Aeronat. Techn. Note 3053.

LLOYD, E. H. (1952), Least-squares estimation of location and scale parameters using order statistics. Biometrika $39,88$.

SARHAN, A. E. (1954), Estimation of the mean and standard deviation by order statistics. Ann. Math. Stat. 25, 317. 